Relations industrielles

Industrial Relations

\title{
The supervision of Personnel, by John Pfiffner, Englewood Cliffs, Prentice-Hall, 1959.
}

\section{C.-R. Giroux}

Volume 15, numéro 2, avril 1960

URI : https://id.erudit.org/iderudit/1022042ar

DOI : https://doi.org/10.7202/1022042ar

Aller au sommaire du numéro

Éditeur(s)

Département des relations industrielles de l’Université Laval

ISSN

0034-379X (imprimé)

1703-8138 (numérique)

Découvrir la revue

Citer ce compte rendu

Giroux, C.-R. (1960). Compte rendu de [The supervision of Personnel, by John Pfiffner, Englewood Cliffs, Prentice-Hall, 1959.] Relations industrielles /

Industrial Relations, 15(2), 277-278. https://doi.org/10.7202/1022042ar

Tous droits réservés (C Département des relations industrielles de l’Université Laval, 1960
Ce document est protégé par la loi sur le droit d'auteur. L’utilisation des services d'Érudit (y compris la reproduction) est assujettie à sa politique d'utilisation que vous pouvez consulter en ligne.

https://apropos.erudit.org/fr/usagers/politique-dutilisation/ 
phases de l'orientation professionnelle consiste à fournir aux personnes intéressées des informations précises sur les groupes d'occupations et sur la nature des occupations. D'où, la nécessité d'organiser un centre de dissémination de l'information, qui reste à la fois assez complet et flexible. Le présent ouvrage a justement comme but d'aider l'orienteur professionnel dans l'aménagement d'une bibliothèque ou centre d'informations sur les occupations.

Cette troisième édition a bien évolué depuis la première et même la deuxième édition. Après un chapitre sur l'utilité et la nécessité des informations occupationnelles, l'auteur fait un exposé démographique du milieu du travail: accroissement et distribution de la population, des employés industriels, structure des occupations, opportunités, etc. Ensuite, lauteur considère les diverses occupations dans leur contexte social. Il est intéressant de noter que la valeur sociale ou le prestige d'une position n'est pas déterminé nécessairement par le contenu de la position ou par sa responsabilité sociale.

Peu de changements ont été apportés au chapitre exposant les diverses méthodes utilisées dans l'analyse et la description des positions. Il aurait été intéressant d'y trouver d'autres méthodes d'analyse des tâches.

Un bref chapitre traite des exigences des positions. Les exigences d'embauchage quoique déterminées par l'employeur sont souvent à la merci des conditions économiques et du marché du travail. Shartle décrit brièvement les examens utilisés pour vérifier si l'applicant rencontre les exigences de la position: examens théoriques, tests psychologiques, etc.

Quoiqu'il n'y ait pas de chapitre spécialement réservé à l'organisation d'une bibliothèque et d'un centre d'informations, il $y$ a suffisamment de suggestions dans le texte pour guider le conseiller d'orientation. Shartle cite de nombreuses sources d'où il est possible d'obtenir de l'information occupationnelle.

Par suite de l'importance croissante des carrières militaires, l'auteur leur consacre tout un chapitre.
Shartle a retenu l'orientation générale du volume, mais il en a amélioré le contenu, en tenant compte des exigences sociales et économiques actuelles.

\section{C.-R. GrRoux}

The supervision of Personnel, by John Pfiffner, Englewood Cliffs, PrenticeHall, 1959.

Quel lecteur l'auteur veut-il atteindre avec ce volume?

Le contenu du volume est beaucoup trop simplifié pour s'adresser à des universtitaires «graduate students 》. D'autre part il est trop compliqué pour les contremaîtres et les surveillants car Pfiffner y traite de validité, fiabilité, corrélations et même d'analyse factorielle. Les administrateurs du personnel et les collégiens «undergraduate students » comprendront assez bien les problèmes qui y sont exposés.

La thèse principale de l'auteur est celle-ci: «Irrespective of class, casts, or occupation, both as individuals and groups, people reacts similarly to particular types of management situations » - En d'autres termes, devant certaines situations les gens réagissent de façon semblable par conformisme. Pfiffner a été fortement influencé par les expériences de Hawthorne et par les recherches de l'Université du Michigan. Tout au long du volume, l'auteur sou tien sa thèse en proposant de tout faire pour maintenir et même si possible augmenter le moral des employés. Sa définition opérationnelle du moral telle que donnée par l'auteur, est fortement discutable car on peut déduire qu'elle prône la manipulation des employés pour atteindre les buts de l'organisation.

«High morale is a complex combination of many factors that make people do what the organization expects them to do. Inversely, low morale is a combination of factors that prevent or deter people from doing what the organization expects them to do ». Pour élever le moral, le contremaître doit appliquer la technique non directive de la méthode clinique. Il doit s'occuper de chaque employé individuellement. Aussi, il faudrait que le contremaître soit entraîné à utiliser la méthode clinique, à diagnostiquer les 
cas de maladies mentales. Pfiffner va jusqu'à soutenir que le contremaître devrait être capable de régler bien des cas personnellement. Quand on songe aux nombreuses années d'entraînement et d'expérience qu'il faut pour développer un thérapeute compétent, il semble que Pfiffner fait preuve d'un idéalisme béat. - D'ailleurs, si le contremaître passe la majeure partie de son temps en entrevue, quand pourrat-il organiser, planifier, et contrôler la production de son département? Quand il est question de «Guidance 》 et de «Counseling », il vaut mieux s'en remettre malgré tout à la compétence professionnelle. L'auteur consacre plusieurs chapitres aux responsabilités cliniques du contremaître.

Comme la majorité des chercheurs Pfiffner ramène la motivation des employés au moral et à plusieurs facteurs de moral qui sont extrinsèques au travail lui-même. Selon lui la participation des employés à la solution de divers problèmes demeure une des plus grandes forces motivantes. Sur ce point nous ne sommes pas du tout d'accord. En principe une politique de participation est acceptable et même souhaitable. Cependant, si l'on considère la complexité des problèmes résultant de la technologie moderne, et les connaissances requises pour aborder ces problèmes, il devient évident que la participation des employés à la planification et à l'organisation de la production va diminuer constamment. Elle diminuera davantage avec la venue de l'automation.

Pfiffner abonde le problème de la discipline de façon réaliste et pratique. Ce chapitre est probablement le mieux étoffé du volume. Nous sommes d'accord avec Pfiffner pour une approche individuelle aux problèmes du développement de la gérance.

En résumé, ce volume n'apporte rien de neuf. Il y gagnerait énormément à être réduit. Les répétitions sont nombreuses au point que plusieurs lecteurs n'auront pas le courage d'en terminer la lecture.

\section{C.-R. GrRoux}

Le syndicalisme francais dans la Ve rémublique, par Raymond Le Bourre, Calmann-Levy Editeurs, 3, rue Auber, Paris, 1959, 212 pp. 606 francs.
La maison Calmann-Levy vient d'éditer une excellente brochure où Raymond Le Bourre tente de faire le point pour le monde syndical français. Il est vrai que cette publication s'adresse plus directement aux milieux ouvriers de la France. Néanmoins, elle ne manquera pas d'attirer l'attention de ceux qui se préoccupent de l'histoire des mouvements ouvriers à l'étranger, d'abord, par son actualité, et en second lieu, par sa présentation.

« Le syndicalisme français dans la Ve république » est une appréciation toute fraîche d'événements très récents que le recul du temps permettra peut-être de mieux évaluer. Cette appréciation est présentée dans un style vigoureux, agressif, souvent de combat et susceptible de soulever certaines controverses. Pour cette raison, elle comporte parfois des biais et des imprécisions. Ainsi, certaines remarques à l'égard de la «Confédération générale des travailleurs chrétiens » (C.F.T.C.) ou de la «Confédération générale du travail » (C.G.T.) s'expliquent si l'on considère que l'auteur n'est pas exempt de préjugés, ayant été membre du parti communiste avec lequel il a définitivement rompu et ayant été l'un des artisans de la scission de 1947 qui donna naissance à la « Force ouvrière 》 (C.G.T.-F.O.) dont il est le secrétaire général depuis 1952. C'est pourquoi l'auteur est parfoís entraîné à faire l'apologie de la C.G.T.-F.O. et à lui assigner une mission particulière.

C'est l'oeuvre d'un militant syndical, sincère, convaincu et fortement engagé qui se fait tantôt polémiste, tantôt théoricien. A notre point de vue, le théoricien a moins de suocès que le polémiste. Par exemple, le chapitre III intitulé: «Le problème des salaires 》 ressemble à un traité d'économie politique miniature et nuit à l'unité logique du volume. On conçoit facilement que l'auteur veut faire une preuve. Mais l'importance qu'il y consacre est en dehors des limites de la thèse qu'il ooutient.

Par contre, l'auteur réussit beaucoup mieux lorsqu'il s'attaque aux problèmes qui confrontent le monde ouvrier. Le chapitre II, « force et faiblesse du mouvement syndical 》 et le chapitre IV, économique \ sont des plus intéressants et démontrent un effort concret de pen- 